\title{
Benign Diseases of the Colon and Rectum (incl. CT colonography)
}

\author{
Thomas Mang and Philippe Lefere
}

\section{Learning Objectives}

- To illustrate the spectrum of benign conditions of the colon and rectum, seen at CT colonography and conventional cross-sectional imaging

- To learn how to characterize and differentiate benign polypoid and stenotic filling defects with 3D and 2D CT colonography imaging criteria

- To discuss both specific and unspecific cross-sectional imaging criteria of various infectious and noninfectious inflammatory conditions of the colon

\subsection{Introduction}

Diagnostic imaging of the colon and the rectum has undergone a remarkable evolution over the last few decades. Routine cross-sectional imaging techniques, such as standard abdominal CT scans, provide the possibility to evaluate the course of the colon for mural and extracolonic changes. With the introduction of CT colonography (CTC) to the clinical routine, a noninvasive and safe diagnostic test for imaging the endoluminal aspect of the entire colon became available. CTC has been shown to be as accurate as optical colonoscopy (OC) for the detection of advanced colonic neoplasia, including advanced adenomas and cancers [1]. Since its performance is clearly superior to that of the barium enema, it is now recommended as the radiological method of choice for the detection of colorectal neoplasia [2, 3]. The barium enema is no longer recommended for this indication. Indeed, the role of the barium enema in daily radiological

T. Mang

Department of Biomedical Imaging and Image-guided Therapy,

Medical University of Vienna, Vienna, Austria

P. Lefere $(\square)$

Department of Radiology, AZ Delta, Roeselare, Belgium imaging has been fading over the last several years due to the emerging use of endoscopic techniques. Today, OC represents the diagnostic and therapeutic gold standard in colonic imaging. CTC has become a valuable complementary diagnostic tool to OC, serving as an alternative colonic screening test, and it represents the method of choice in patients with incomplete colonoscopy or with contraindications to colonoscopy [3].

The purpose of this contribution is to focus on benign conditions of the colon and rectum that can be detected during CTC and routine cross-sectional imaging techniques.

\subsection{Benign Colonic Findings Seen at CTC}

\subsubsection{Benign Mucosal Colonic Polyps}

Polyps are the most common benign lesions of the colon. In addition to malignant tumors, polyps are the main targets of CTC. According to the Paris classification, they are categorized by their morphologic appearance as sessile, pedunculated, or flat [4]. On CTC, polypoid findings of the colon are characterized by their outer morphology, their internal structure and attenuation characteristics, and their mobility between the supine and prone patient positions [5].

A sessile polyp (0-Is) presents as a round, oval, or lobulated intraluminal filling defect, with a base that is equal to or larger than its vertical height (Fig. 19.1).

A pedunculated polyp (0-Ip) presents with a round, oval, or lobulated polyp head that is connected to the mucosa by a stalk. On 2D planar images, colorectal polyps typically show a homogeneous internal structure with a soft tissue attenuation. Polyps arise from the colonic wall. Therefore, they maintain their intraluminal position when the prone and supine scanning positions are compared. This criterion may help to differentiate true polyps from untagged residual stool that is not attached to the colonic wall and that shows a positional movement. Pedunculated polyps, however, may show a 

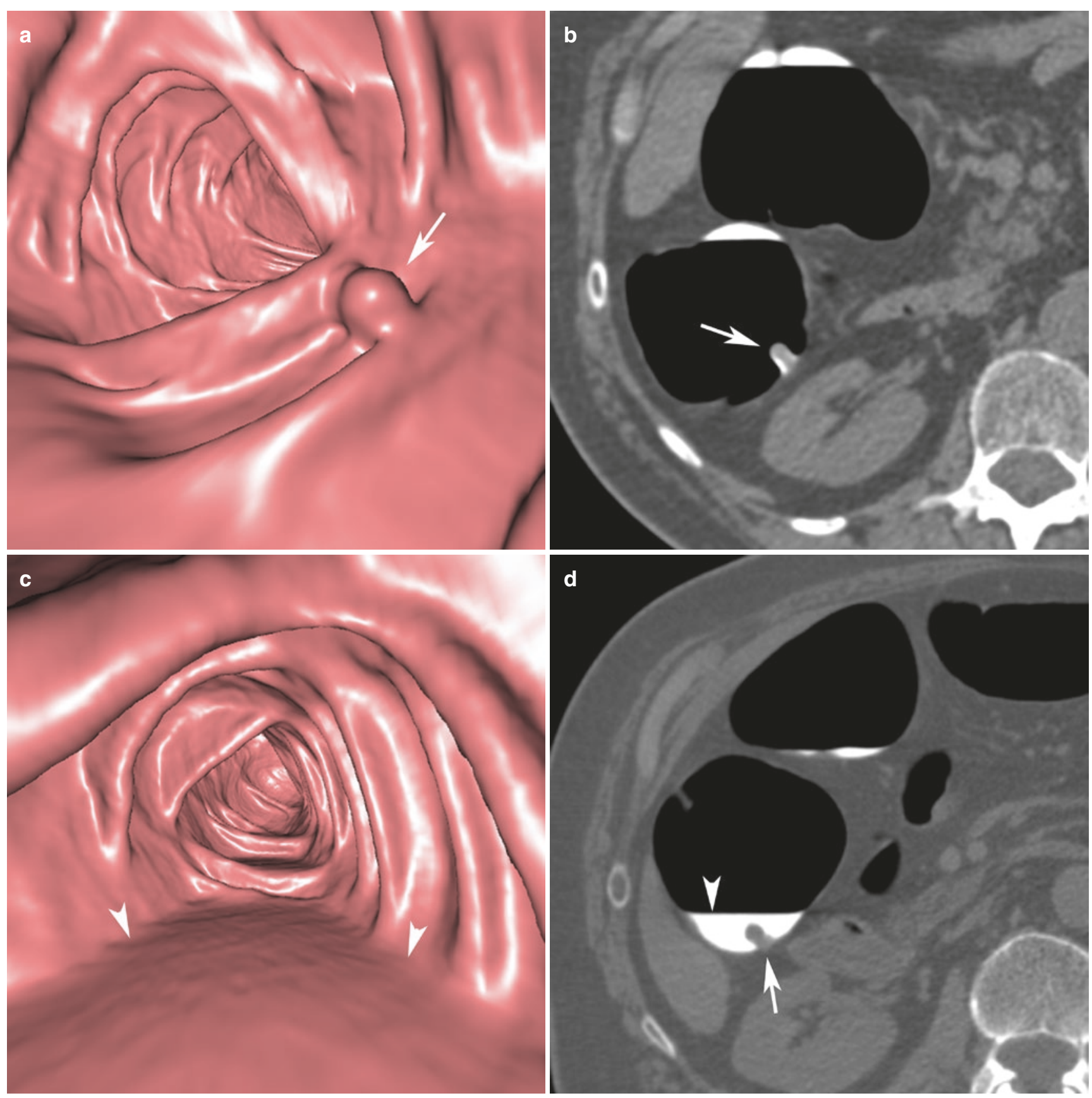

Fig.19.1 (a-d) Sessile oval polyp located in the ascending colon. (a) The endoluminal 3D view shows a round polypoid lesion (arrow) (b) The corresponding prone axial 2D image shows the homogeneous softtissue density (arrow) of the lesion. (c) The corresponding supine endo-

considerable positional shift between the supine and prone acquisitions because of their stalk (Fig. 19.2). This so-called pseudo mobility may cause confusion with the untagged stool, showing true mobility, i.e., a pseudo-stool appearance.

Flat or non-polypoid lesions are morphologically characterized by their low elevation compared to their width (Fig. 19.3). On CTC, non-polypoid lesions are defined as lesions that measure $6 \mathrm{~mm}$ or larger above the surrounding mucosa, with a height of $3 \mathrm{~mm}$ or less [6]. Endoscopically, they are categorized luminal 3D view shows a horizontal fluid layer (arrowheads). No polyp is seen. (d) The supine axial 2D image shows that the lesion (arrow) is submerged unter tagged residual fluid (arrowhead)

according to their predominant direction of growth, which defines their morphology: flat elevated (0-IIa), truly flat (0-IIb), and depressed flat (0-IIc). Non-polypoid lesions are generally less common than their polypoid counterparts.

On CTC, they are less conspicuous than polypoid lesions. On endoluminal 3D views, flat lesions present as a plaquelike elevation of the colonic wall, with a smooth or nodular surface. A flat lesion, located on a semilunar fold, typically leads to a circumscribed thickening of the fold. On 2D pla- 

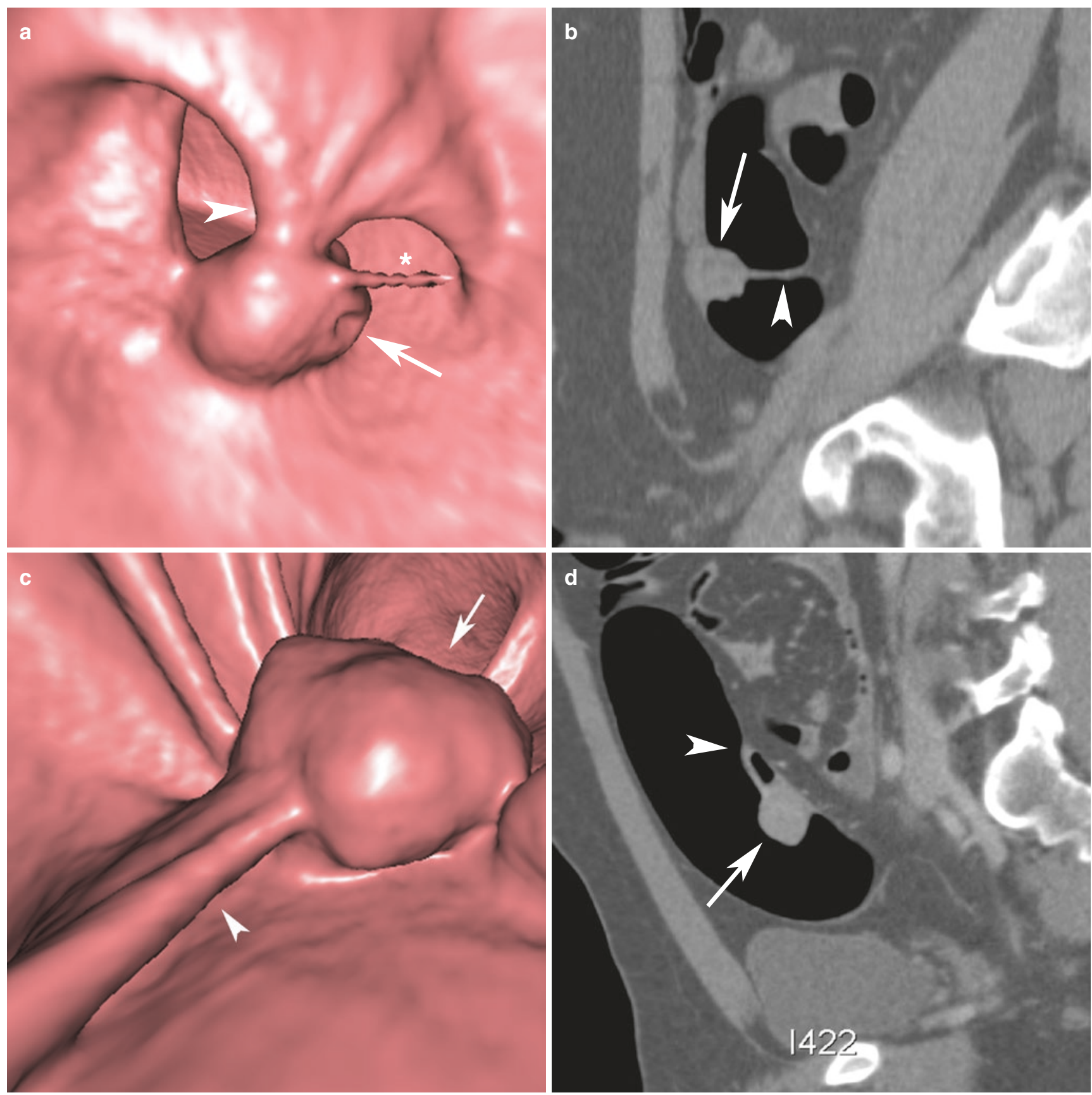

Fig. 19.2 Large pedunculated polyp located in the sigmoid colon. (a) The prone endoluminal 3D view shows the polyp head (arrow), connected to the mucosa by its stalk (arrowhead). A small mucus tear is attached to the polyp head (asterisk). (b) The corresponding prone sagittal 2D image shows the soft tissue dense polyp head (arrow) located

nar views, flat lesions typically appear as circumscribed, plaque-like wall thickening with a homogeneous soft-tissue attenuation [5]. Flat lesions with a central depression have been shown to have a higher risk for malignancy. Flat lesions are frequently covered by tagged residue, which may aid detection. This is called "contrast coating" [7].

Larger non-polypoid tumors, spreading over $\geq 1 \mathrm{~cm}$ (on CTC $\geq 3 \mathrm{~cm}$ ), are laterally spreading tumors (LSTs). An

on the anterior sigmoid wall. It is connected by the stalk (arrowhead) to the dorsal sigmoid wall. (c) The corresponding supine endoluminal 3D view and (d) sagittal 2D image show that the polyp head (arrow) moves on its stalk (arrowhead) to the dorsal sigmoid wall

older, commonly used term is a "carpet lesion." They are an important subset of flat lesions, with a predilection for the cecum and the rectum. They typically present with advanced histological features. Based on their surface appearance, they are endoscopically categorized as granular or non-granular types [8].

On CTC, they typically present with a broad-based flat morphology, with a size $\geq 3 \mathrm{~cm}$. They may exceed $3 \mathrm{~mm}$ in 


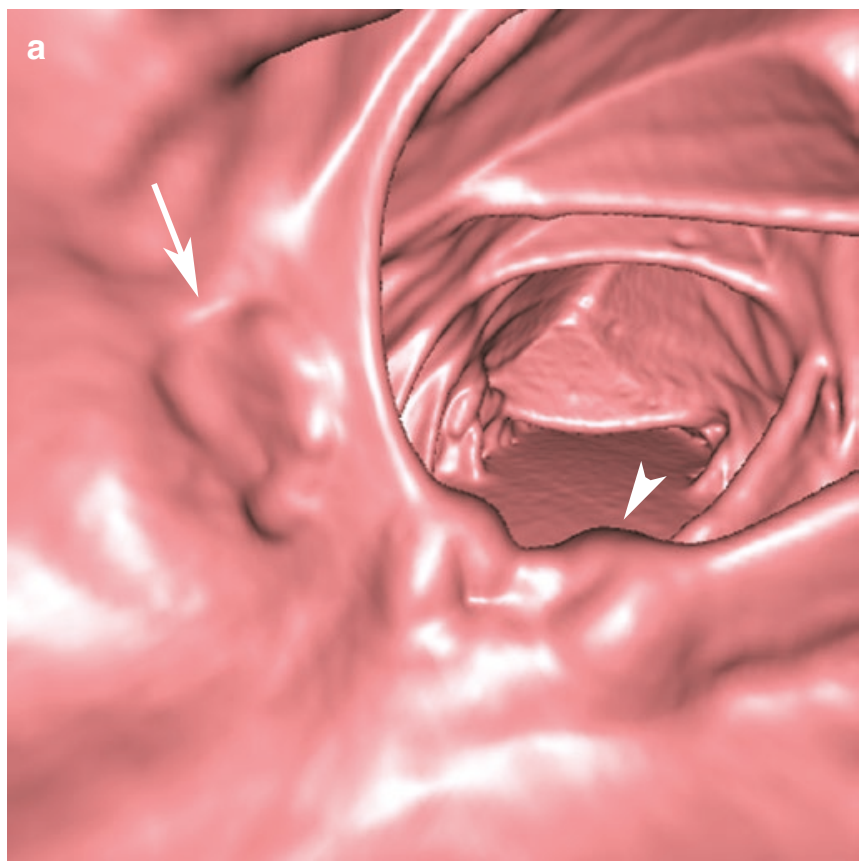

Fig. 19.3 (a, b) A $12 \mathrm{~mm}$ slightly elevated flat lesion with a central depression. (a) The endoluminal 3D image shows a flat slightly elevated filling defect with a central depression (arrow), located on a haustral fold adjacent to the ileocecal valve (arrowhead). (b) The

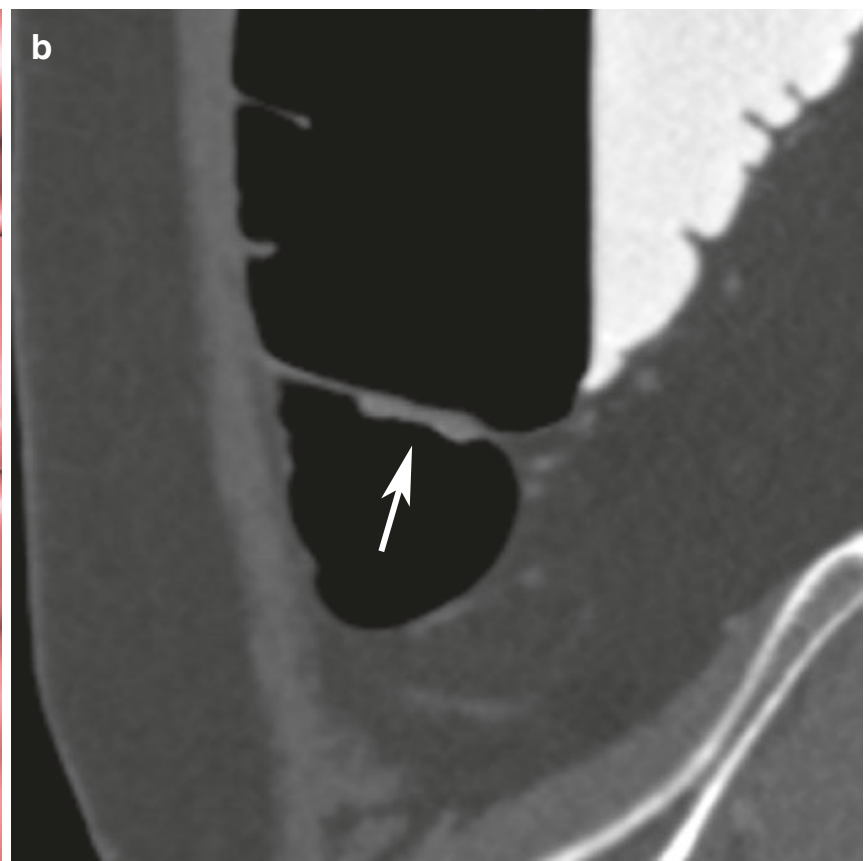

corresponding sagittal 2D view shows a flat elevation of the bowel wall leading to a plaque-like soft tissue thickening of the haustral fold. Note the slight central depression at the center of the lesion (arrow)

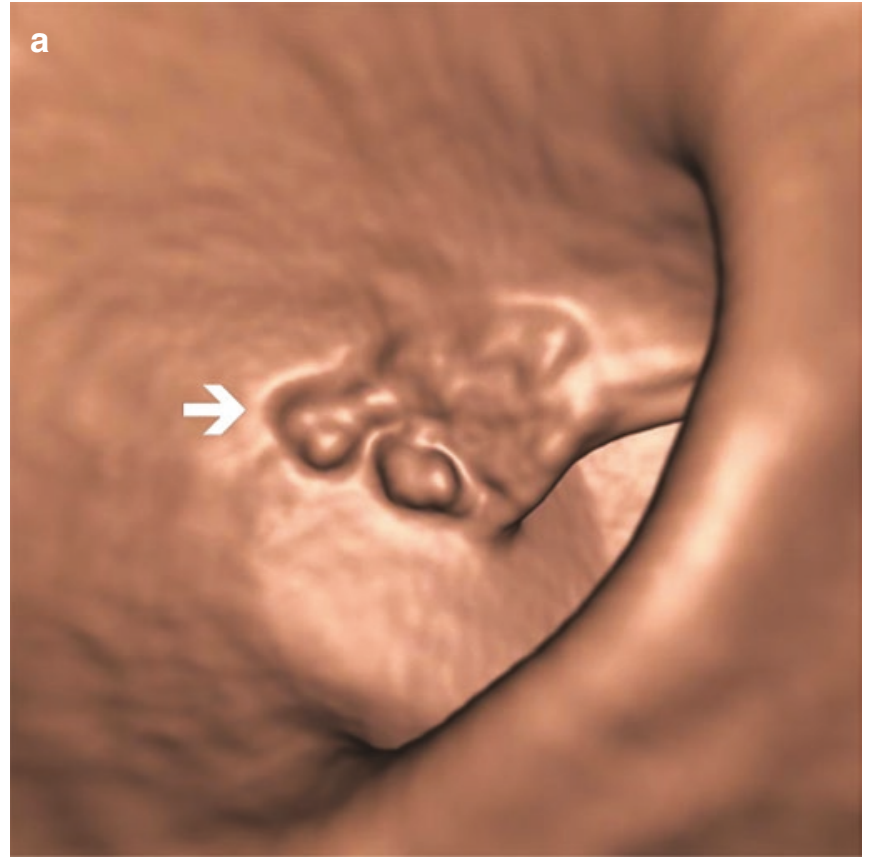

Fig. 19.4 (a, b) Laterally spreading tumor of the mixed granular type in the rectum. (a) The endoluminal 3D view shows a flat irregular filling defect with a nodular surface (arrow). (b) The corresponding axial 2D

height but are still flat and may have superficially raised edges. Surface coating with oral contrast material is frequently seen [9] (Fig. 19.4).

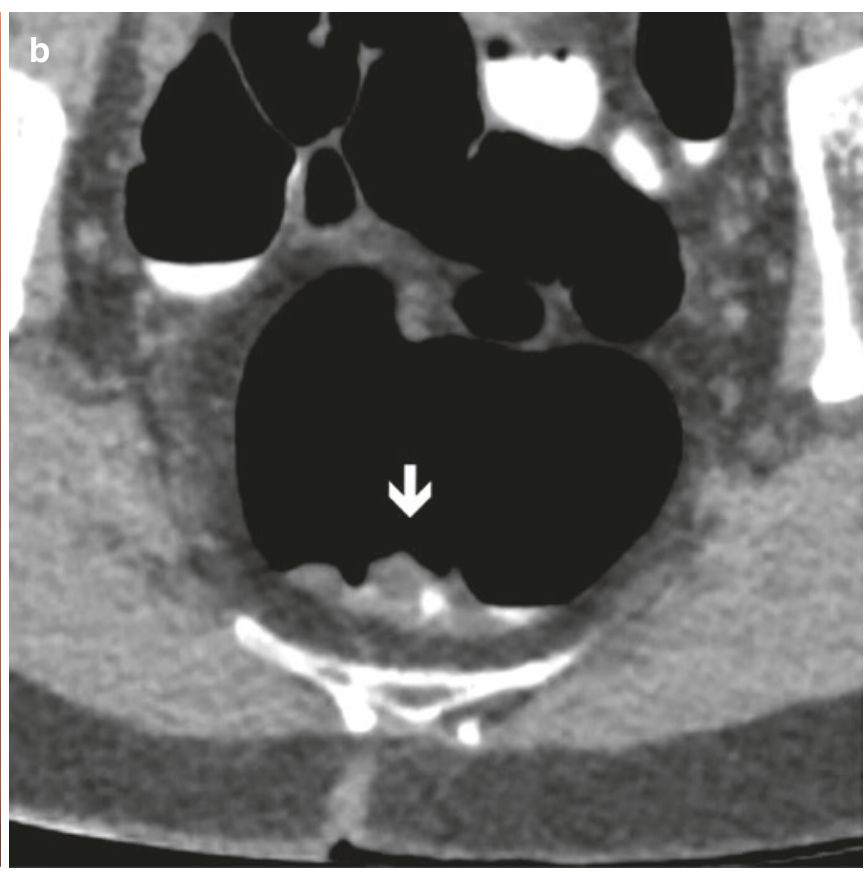

image shows a nodular wall thickening with soft tissue attenuation (arrow). The lesion is covered by a small amount of tagged fluid

Correct CTC size measurement is important because the size of a polyp, as measured by the radiologist, has a direct influence on the selection of the appropriate therapeutic 
procedures. There is general consensus that all polyps measuring $6 \mathrm{~mm}$ or larger need to be reported, and endoscopic polypectomy should be recommended $[3,6]$. Polyps smaller than $6 \mathrm{~mm}$ are frequently ignored. For sessile and flat polyps, the greatest diameter is measured, which is most often at the base of the lesion. For pedunculated lesions, only the largest diameter for the polyp head should be measured, excluding the stalk [6].

\subsubsection{Benign Submucosal Tumors}

\subsubsection{Lipomas}

Lipomas are the most common submucosal tumors in the colon. They are typically between 1 and $3 \mathrm{~cm}$ in size. A lipoma can have a sessile, pedunculated, or even flat morphology. On endoluminal 3D views, they present as filling defects, typically with a smooth surface. A correct diagnosis is obtained with 2D views: lipomas typically have a homogeneous fatty attenuation. Often, a thin mucosal stripe covering the fatty tissue is seen. Since lipomas are relatively soft lesions, they may change their shape when the position of the patient is changed (Fig. 19.5). Lipomas are benign. No diagnostic or therapeutic investigation is needed. Large lipomas, however, can be symptomatic and lead to intussusceptions [10].

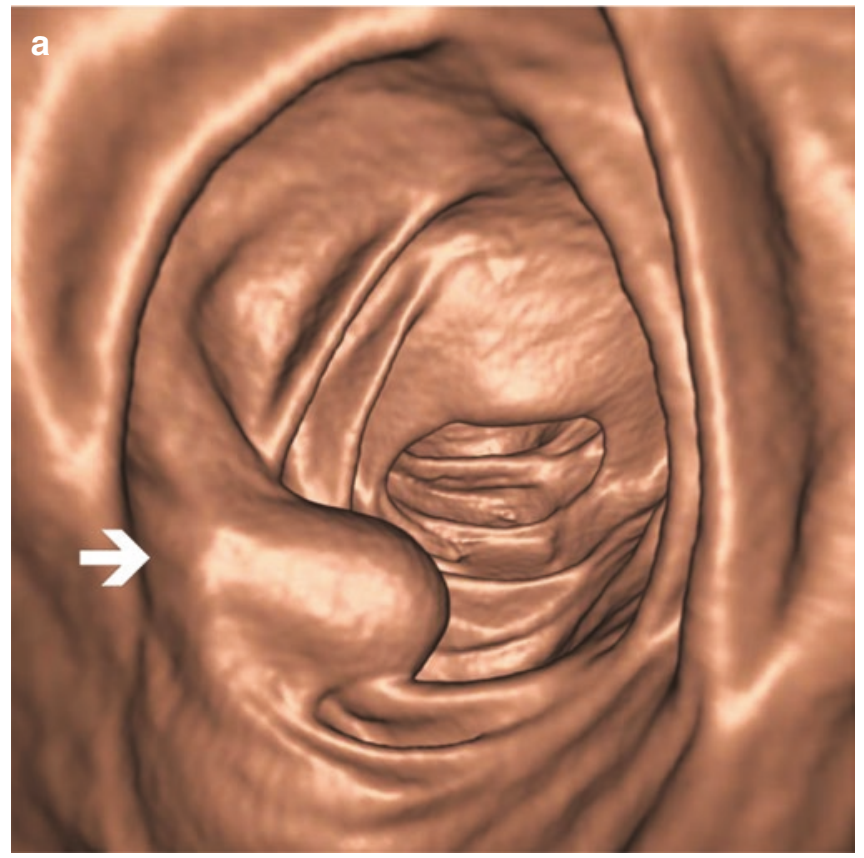

Fig. 19.5 (a, b) Lipoma in the ascending colon. (a) The endoluminal $3 \mathrm{D}$ view shows a large polypoid lesion with smooth appearance (arrow). (b) The corresponding axial 2D image shows the homogeneous fat tis-

\subsubsection{Rare Submucosal Tumors}

Lymphangiomas are rare benign lesions which may be simple, cavernous, or cystic. In case of the cystic type, the lesion appears with a cystic morphology on 2D views. However, whenever the finding is $\geq 6 \mathrm{~mm}$ on $\mathrm{OC}$, it is necessary to exclude a true neoplastic lesion. There is a wide range of other submucosal tumors that are typically very rare, including leiomyoma, hemangioma, and ganglioneuroma. These cannot be differentiated from adenomatous polyps or tumors based on CT imaging criteria [10].

\subsubsection{Pneumatosis Cystoides Coli}

Pneumatosis coli is a rare entity with intramural collections of gas. The primary type is benign and self-limiting. The secondary type is related to bowel wall necrosis. It usually requires immediate treatment according to the clinical presentation. On CTC, the primary type has been reported as a rare finding. Primary pneumatosis coli presents with pseudopolypoid filling defects on endoluminal $3 \mathrm{D}$ views, corresponding to submucosal cystic or linear pockets of air. Two-dimensional views reveal the pathognomonic air attenuation on these findings. Primary pneumatosis coli is a totally innocuous, asymptomatic, and self-limiting "complication," which requires no further medical action [11].

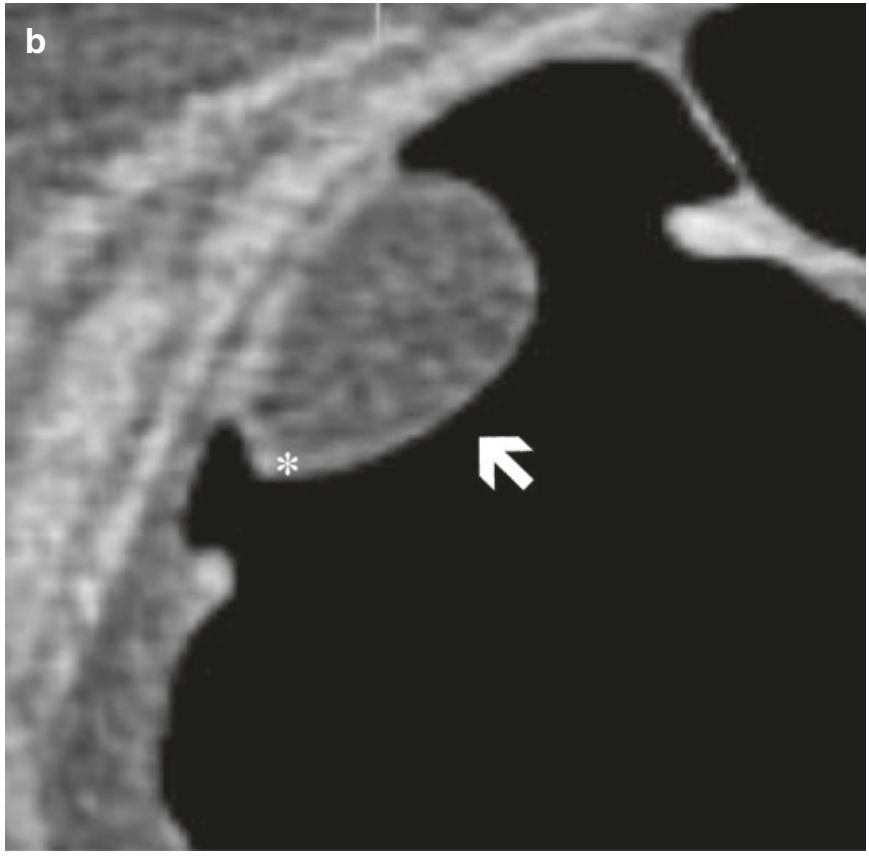

sue density (arrow) of the lesion, covered by a thin mucosal stripe confirming its submucosal origin (asterisk) 


\subsubsection{Endometriosis}

Endometriosis is a condition related to the growth of the endometrium outside the uterus. Colonic involvement occurs mostly in the rectosigmoid and rarely in the cecum. This occurs via peritoneal implants of endometrial tissue. These implants may develop trans-serosal growth with submucosal extension. From there, intraluminal extension may occur in a small number of patients. According to the grade of invasion, CTC shows different aspects. With serosal/submucosal infiltration, there is mural thickening, which leads to a marginal luminal filling defect, showing a smooth delineation (Fig. 19.6). In case of extension to the mucosa, the wall becomes irregular and ends in a polypoid lesion or mass [12].

\subsubsection{Anatomy-Related Findings}

\subsubsection{Segmental Colonic Spasm}

Colonic spasm is a physiological narrowing of the intestinal lumen due to muscular contraction of the colonic wall that can simulate circular stenotic tumors. The degree of colonic distension and the intraluminal aspect of the colon also depend on the muscular contraction of the taenia coli. Optimal colonic distension prevents spasms and possible false-positive findings. In the sigmoid, the taenia is more or less subtle. When contracted, they cause the narrowed lumen to be round, with smoothly thickened folds without over- hanging edges. In the descending colon, the taenia becomes slightly more prominent with, in contraction, a slightly more triangular aspect of the colonic lumen. In the transverse and ascending colon, the triangular shape of the lumen is obvious. However, the folds are not distorted, and there is usually no shoulder formation, which is typical of malignancy. Due to redistribution of intracolonic gas, the spasm will frequently either change in morphology or even disappear between the supine and prone acquisitions [5].

\subsubsection{Flexural Pseudotumor}

The colon presents with many loops and flexures. At each flexure, the structure at the inner side is more compressed than that on the outer side. This can cause complex structures that consist of crowded and thickened colonic folds. This thickening is accentuated by the pericolic fat and its vascular structures. On 2D planar views, this may lead to an apparent wall thickening that may be mistaken for a colonic mass. Therefore, such thickening is referred to as a flexural pseudotumor. Such pseudolesions can become real tumor mimics in case of a flexure over an acute angle with the convergence of many folds. A comparison of both acquisitions will help to detect any change in appearance. A thorough analysis of the appearance on 3D and 2D images is helpful to exclude a tumoral process. The pseudolesion most frequently consists of a convergence of many folds that show regular smooth contours on endoluminal 3D views. On $2 \mathrm{D}$ views, pericolic fat is sometimes demonstrated in the thickened fold [13].
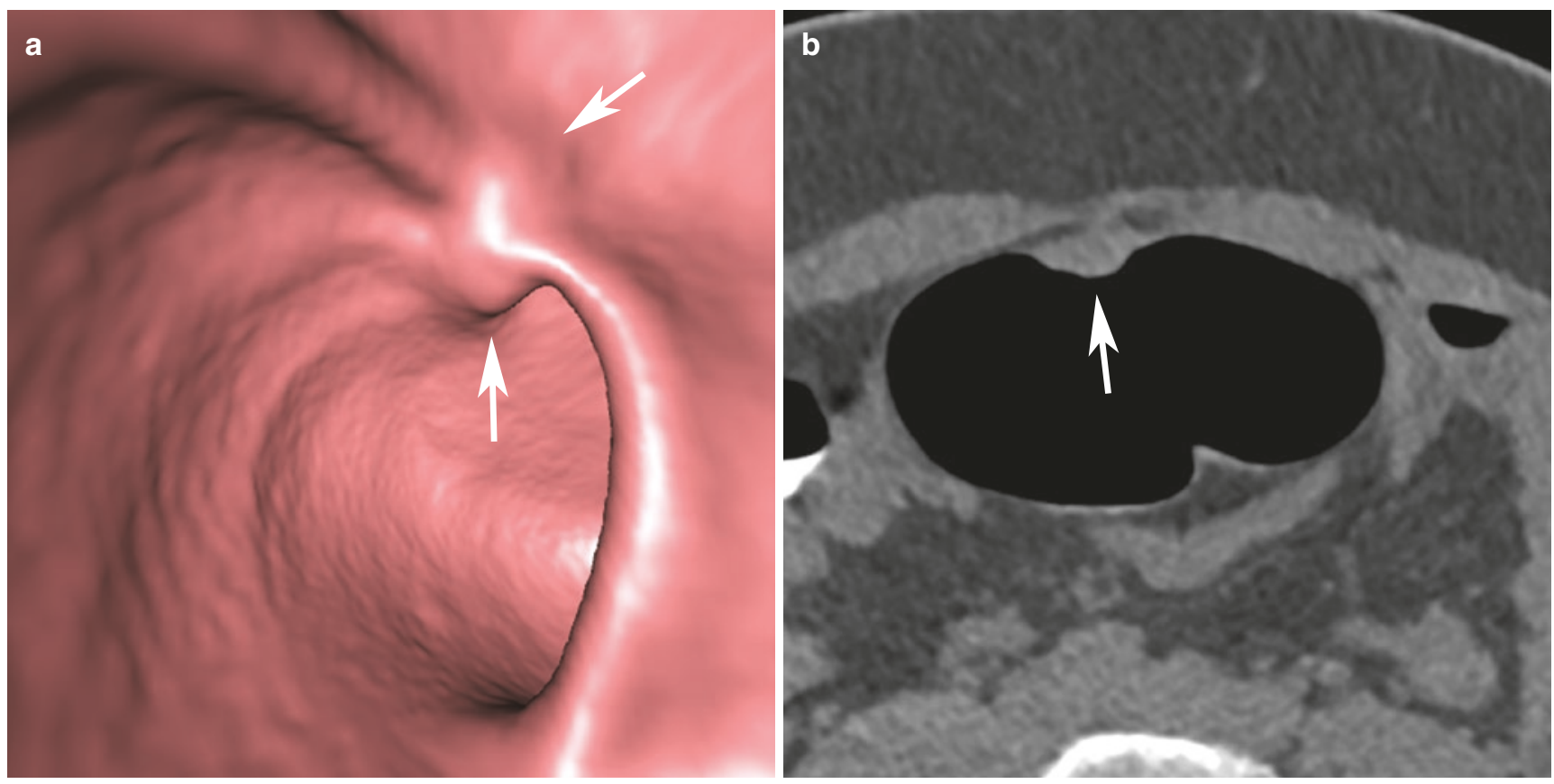

Fig.19.6 (a, b) Endometriosis implant in the sigmoid colon. (a) The endoluminal 3D view shows a flat intraluminal filling defect with a smooth delineation (arrows). (b) The corresponding axial 2D image shows a homogeneous soft tissue dense lesion (arrow) infiltrating the colonic wall 


\subsubsection{The Anorectal Region}

For the evaluation of the rectum, it is important to inspect the anal margin. This is done by turning the virtual camera to the anus. In this part of the rectum, several benign and/or tumorlike conditions are possible $[5,13,14]$.

\subsubsection{The Hypertrophied Anal Papilla}

The hypertrophied anal papilla is a frequent finding. It is a papillary projection of a rectal column (Morgagni) and appears as a fibrous white polyp of any size at OC. On CTC, it is impossible to differentiate from a true polyp, necessitating rectoscopy in case of a polypoid structure $\geq 6 \mathrm{~mm}$.

\subsubsection{Internal Hemorrhoids}

Internal hemorrhoids are varicosities of the superior hemorrhoidal plexus. They arise at the anal margin and are a very frequent finding ( $\pm 50 \%$ of patients). They typically appear as smooth filling defects surrounding the rectal catheter with thin folds converging toward the catheter. On rare occasions, they may have a polypoid appearance.

\subsubsection{Other Findings}

The rectal varix is a dilated submucosal vein that causes a long, serpiginous filling defect. It is important to be aware of its existence, as, on rare occasions, polyps may also have a serpiginous aspect. A venous bleb is a small venous malformation that may present with a polypoid morphology and mimic true polypoid lesions. However, usually they are $<5 \mathrm{~mm}$.

\subsubsection{The Cecum}

The cecum has a complex topography including the ileocecal valve and the appendix. As with the rectum, its CTC examination requires specific attention [15-17].

\subsubsection{The lleocecal Valve}

The normal ileocecal (IC) valve consists of twofolds (upper and lower lip), converging at both sides in a prominent fold, the frenulum. When examining the cecum, it is always essential to identify the IC valve and check its anatomic appearance, excluding polyps and cancers located on the valve. The normal IC valve, however, may show some pseudotumoral aspects that should not be confused with colonic pathology.

\subsubsection{Lipomatous Transformation}

Lipomatous transformation refers to fatty infiltration of both lips of the IC. The IC valve is typically enlarged and may have a dome-like appearance. Correct characterization of this benign anatomical variant is obtained by assessing the typical fat attenuation (0/-100 H.U.) in an abdominal window setting.

\subsubsection{Papillary Transformation}

Papillary transformation is less frequently observed than the normal labial appearance. The papillary IC valve is caused by protrusion of the terminal ileum into the IC valve. This is considered a normal functional status, with the IC valve preventing fluid reflux from the colon into the ileum. A papillary IC valve is recognized by a bulbous or polypoid morphology and a smooth surface. It may also appear slightly distorted and presents as a bulky, mass-like lesion. In such cases, the diagnosis is not always straightforward. Most often, this condition is characterized by a smooth delineation of the IC valve with some fatty infiltration on 2D images. The appearance frequently changes with dual acquisition (i.e., supine-prone). However, any accumulation of soft tissue should raise the suspicion for neoplasia.

\subsubsection{The Appendix}

As the appendix is part of the colon, it is necessary to inspect it. On endoluminal 3D views, the orifice of appendix is identified as a small orifice or depression frequently located in the vicinity of small fold. On 2D planar views the appendix presents as a thin-walled tubular structure, which may be filled with air or tagged fluid. Any global or focal wall thickening should prompt further investigation to rule out malignancy (appendectomy). Tumoral conditions of the appendix are rare and are frequently asymptomatic [18].

\subsubsection{Mucocele of the Appendix}

An appendiceal mucocele corresponds to a mucus-dilated appendix. It typically appears as a tubular cystic lesion in the course of the appendix and may present with a mural calcification. This may correspond to a simple mucocele (retention cyst) or a hyperplastic mucocele (hyperplastic polyp) in $5-20 \%$ of cases, to mucinous cystadenoma in $63-84 \%$, and to mucinous cystadenocarcinoma in 11-20\%. Rarely, it corresponds to endometriosis. A mucocele can protrude into the cecal lumen and presents as a polypoid lesion on endoluminal 3D views (Fig. 19.7) [19]. A malignant cause has to be excluded by surgery.

\subsubsection{Inverted Appendiceal Stump}

Following inversion-ligation appendectomy, which is an older appendectomy technique, an appendiceal stump may be seen as a polypoid lesion in the region of the expected appendiceal orifice. It presents as a round smooth polypoid filling defect at the site of the appendiceal orifice with a homogeneous soft-tissue attenuation. This postsurgical polypoid finding cannot be differentiated from a true colonic polyp based on CT imaging criteria. If there is uncertainty concerning previous appendectomy, OC should be recommended, the same as for any polypoid lesion $\geq 6 \mathrm{~mm}$. Furthermore, on rare occasions, a polyp or malignancy may develop in the stump [20]. 


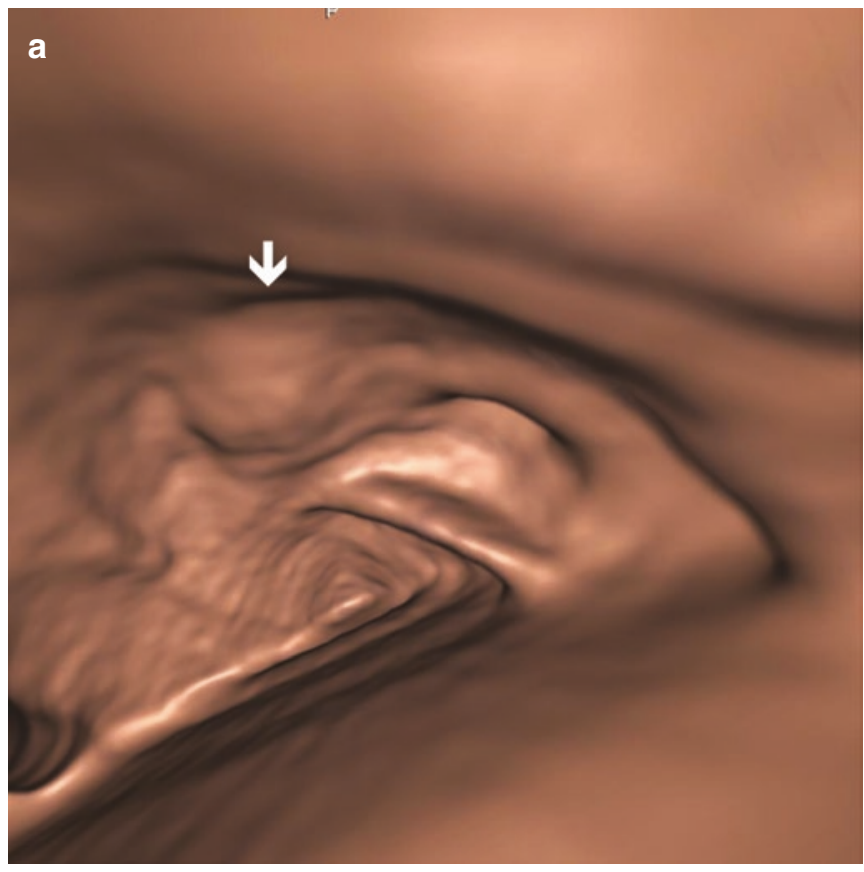

Fig. 19.7 (a, b) Mucocele of the appendix. (a) The endoluminal 3D view of the partially fluid-filled cecum showing a broad-based polypoid intraluminal filling with a smooth surface (arrow). (b) The correspond-

\subsubsection{Diverticular Disease}

Diverticular disease is the most common disease of the colon in the Western population, with a frequency of about $30 \%$ at age 50 and more than $50 \%$ at age 60 . It is mostly located in the sigmoid colon. In its early stage, diverticular disease is characterized by myochosis: a triad of muscular wall thickening, shortening of the taenia, and luminal narrowing. This results in restricted colonic distensibility with prominent and narrow stranding semilunar folds and deep haustrations, with shortening of the interhaustral segments (concertina appearance). On endoluminal 3D views, there is restricted mucosal visualization behind and between the prominent semilunar folds (blind spots). Dedicated 3D inspections are required, which involve turning the virtual camera around to visualize the backside of the folds and the interhaustral segment. To improve colonic distension, the use of hyoscine- $N$ butylbromide is justified [6]. Distension can also be improved by repositioning the patient to the right or left lateral decubitus position, with an additional low-dose CT scan of the affected segment.

With progression of myochosis, the diverticula appear and the semilunar folds become more prominent. On 2D images, the diverticula are characterized by gas-filled outpouchings on the colonic wall. On endoluminal 3D views, they appear as a well-defined complete dark ring that may simulate a small sessile polyp lesion. Polyps, in contrast, often present with an incomplete or vague ring shadow when

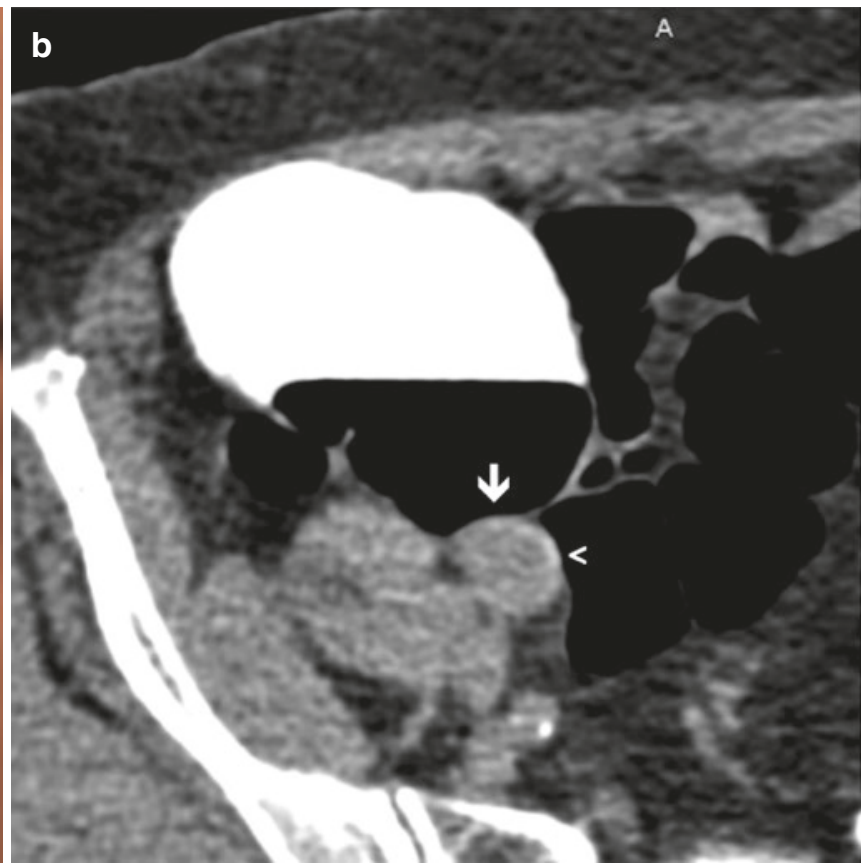

ing axial 2D image shows a cystic structure, causing a subtle extrinsic impression on the colonic lumen (arrow). The lesion had a thin mural calcification (open arrowhead) and was contiguous with the appendix

viewed en face. Two-dimensional views make differentiation of diverticula and polyps straightforward. Diverticular disease gives rise to pseudopolypoid lesions [21].

\subsubsection{The Diverticular Fecalith}

Diverticula are often impacted with fecal residue that can protrude through the neck of the diverticulum into the colonic lumen. This causes a polypoid filling defect on endoluminal $3 \mathrm{D}$ views, with a pathognomonic aspect on $2 \mathrm{D}$ views: the diverticular fecalith almost invariably presents as a round structure with a hyperdense ring and a hypodense center corresponding to an air occlusion [22]. It can protrude from the diverticulum into the colonic lumen. Diverticular fecaliths can be the source of inflammation that leads to focal diverticulitis.

\subsubsection{The Inverted Diverticulum}

A diverticulum may invert into the colonic lumen. Although infrequent, radiologists need to be aware of this possibility. On endoluminal 3D views, the inverted diverticulum mimics a true polyp with, on 2D planar views, mostly an air or fat inclusion. Sometimes, a central umbilication can be distinguished.

\subsubsection{Chronic Diverticular Disease or Stenosing Cancer}

Patients with repetitive episodes of acute diverticulitis may develop chronic diverticular disease, a common reason for 
Table 19.1 CT colonography imaging characteristics suggestive of chronic diverticular disease in the left column, and a stenosing colonic cancer in the right column

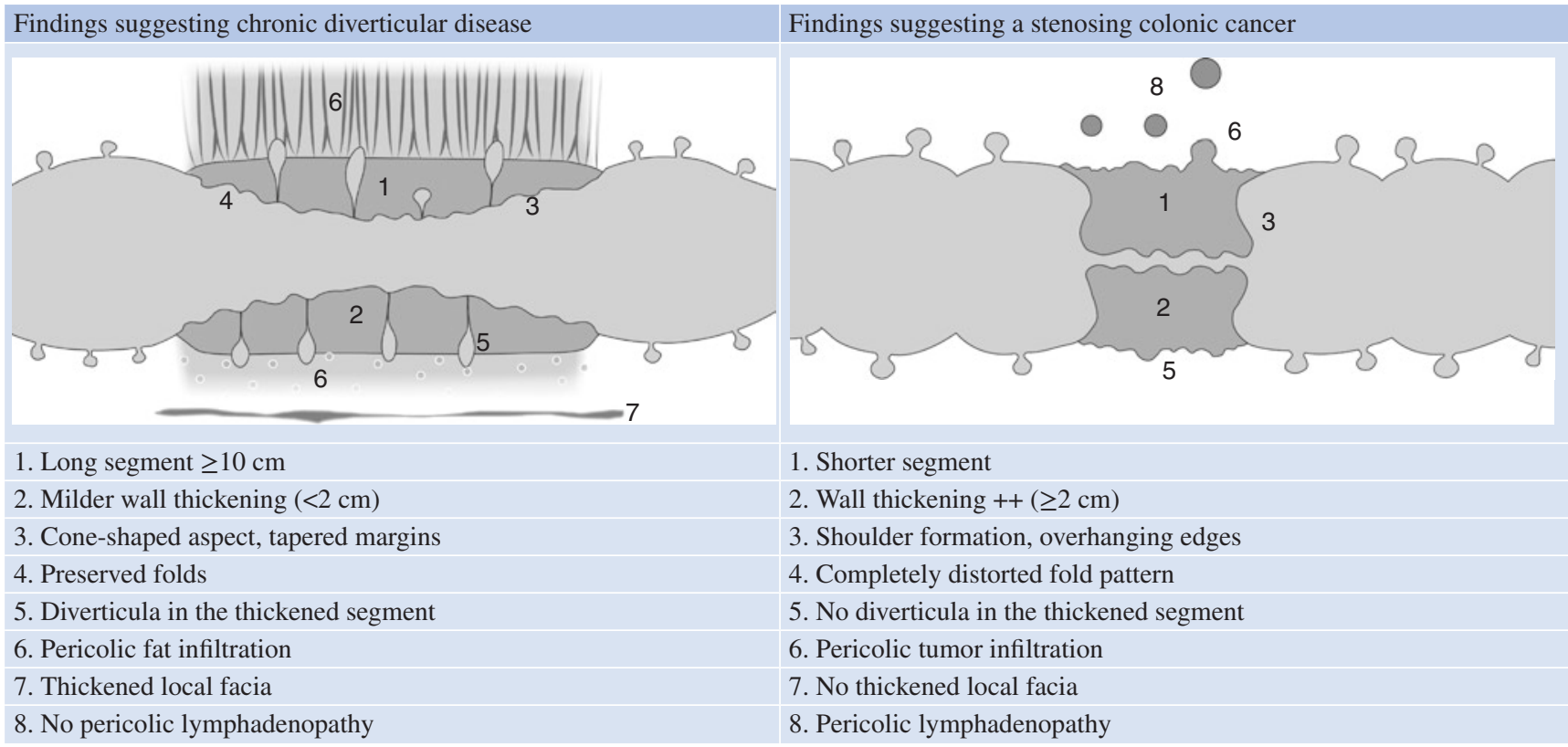

Note that these signs overlap each other, making an exact diagnosis difficult and sometimes impossible. However, the absence of diverticula within the affected segment and the presence of shoulder formation are strong indicators for cancer

incomplete OC. CTC is the method of choice to complete the colonic evaluation. However, differential diagnosis between chronic diverticular disease and a stenosing cancer can be challenging. Each entity presents with imaging characteristics which, unfortunately, are not entirely specific. Interference of signs is quite frequent. Features, characteristic of chronic diverticular disease and malignant tumor, are presented in Table 19.1. Studies comparing both entities concluded that it seems reasonable that the presence of diverticula in the affected segment, together with wall thickening over a rather long segment, thickening of the local fascia (fascia sign) without lymphadenopathy, cone-shaped or tapered margins, and the preserved more or less thickened colonic folds, is likely indicative of chronic diverticular disease. Conversely, the absence of diverticula in the thickened segment and thickening of the local fascia, a more prominent wall thickening with a short extension, shoulder formation or overhanging edges, and a completely distorted or fold pattern are more likely indicative of a stenosing colonic cancer $[23,24]$.

\subsubsection{Postoperative Colon}

According to the surgical technique used (end-to-end or side-to-end), the anastomosis will have a different morphology. The anastomosis should show a smooth transition, with, occasionally, a subtle, smooth, circumferential ridge, or a web-like appearance. On 2D views, the surgical staples, if used, are easily identified. Sometimes, the staples protrude into the colonic lumen, causing a tiny luminal filling defect on 3D views that can be easily identified on $2 \mathrm{D}$ views by its high internal density. Polypoid granulation tissue as well as inflammatory and hyperplastic polyps may develop at the anastomosis. These findings will present with the same imaging features as adenomatous polyps. In the postoperative period, fibrosis may develop, causing a pseudotumoral narrowing at the anastomosis [25]. Therefore, any increase of soft tissue at the site of, or adjacent to, the anastomosis should raise the suspicion for tumor recurrence, indicating OC.

\subsection{Inflammatory Diseases of the Colon}

In addition to benign polypoid and stenotic findings and their pitfalls, several other benign colonic conditions may be encountered with diagnostic imaging techniques. They include a wide range of inflammatory diseases, including diverticulitis, chronic inflammatory bowel diseases (CIBDs), infectious and noninfectious colitis, as well as mechanical colonic obstruction and perforation. In almost all of these acute conditions, CTC is contraindicated since the distension of the colon with gas may lead to colonic wall perforation. For these indications, standard cross-sectional imaging is the method of choice. Furthermore, some of these entities require histologic workup to achieve a correct diagnosis or identification of dysplasia. 
In the large bowel, inflammation typically leads to bowel wall thickening, with or without mural stratification, which is the ability to distinguish the different layers of the colonic wall on crosssectional imaging [26, 27]. Various inflammatory bowel diseases differ in their primary location within the GI tract, length of segmental involvement, degree of wall thickening, mural enhancement pattern, and extraintestinal involvement.

\subsubsection{Diverticulitis}

Inflammation of diverticula leads to symptomatic diverticulitis, which occurs, in two-thirds of cases, in the sigmoid colon. This can affect a single or a few adjacent diverticula, called focal diverticulitis, or the entire colonic segment. If a single diverticulum is inflamed, there is focal, edematous mural thickening, typically surrounding a stool-impacted diverticulum, with increased mural enhancement and focal pericolonic fat stranding [28]. Imaging findings of acute colonic diverticulitis are cone-shaped inflammatory wall thickening, involving a long segment $(>10 \mathrm{~cm})$, and increased mural contrast enhancement, as well as submucosal edema. Acute inflammation is typically accompanied by pericolic fat stranding and fluid at the root of the mesentery (Fig. 19.8). The presence of diverticula is pathognomonic. Their absence makes diverticulitis unlikely [29]. Complications that may develop are pericolic abscess, contained or free perforation, hemorrhage, fistula formation, and post-inflammatory stenosis. The most impor-

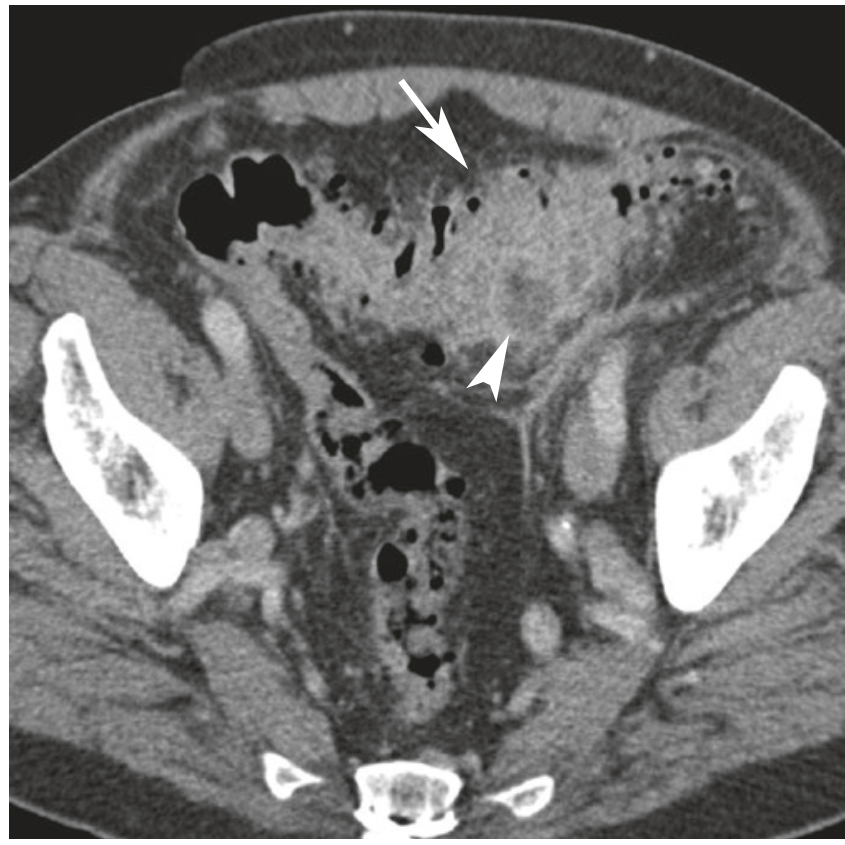

Fig. 19.8 Acute diverticulitis. The contrast-enhanced axial CT image shows wall thickening of the sigmoid colon (arrow) with adjacent inflammatory stranding of the pericolic fat tissue. Note the fluid collection representing abscess formation (arrowhead) tant differential diagnosis for diverticulitis is colon cancer. In contrast, extensive wall thickening with short extension $(<5 \mathrm{~cm})$, especially with shoulder formation and pericolonic lymph nodes, is suspicious for neoplasms [30].

\subsubsection{Colonic Involvement in Chronic Inflammatory Bowel Disease}

Within the group of CIBD, Crohn's disease (CD) and ulcerative colitis (UC) represent the most important conditions. OC with biopsy is the examination of choice to evaluate colonic involvement in CIBD, specifically for the detection of colonic dysplasia, and to differentiate between an inflammatory stenosis and cancer. Standard abdominal CT is indicated for the detection of acute complications. CTC is not indicated and may be an option only in patients with incomplete OC in chronic inactive disease.

\subsubsection{Ulcerative Colitis}

Ulcerative colitis is an inflammatory bowel disease limited to the mucosa and submucosa of the colon. The disease typically begins in the rectum and continuously extends proximally to involve parts of the colon or the entire colon. In $10-40 \%$ of cases, the distal ileum is also inflamed, which is referred to as backwash ileitis.

Early, subtle, and inflammatory mucosal changes, such as the granular pattern of the mucosa or tiny punctuate ulcers, may be beyond the current spatial resolution of modern cross-sectional imaging.

Progression of the disease leads to mucosal hyperemia and submucosal edema, which then result in thickening and stratification of the wall, accompanied by increased pericolic vascularity. Increased ulceration and pseudopolyps appear, and the mucosa becomes friable. Typically, the outer contour of the affected colonic wall is regular and smooth (Fig. 19.9). Lymph node enlargement is only slight. The appearance of abscess or fistula formation is uncommon.

Subacute and chronic forms lead to thickening and rigidity of the wall. Narrowing of the colonic lumen and foreshortening of the colon may occur. The bowel loses its haustral pattern, which can result in a tubular "lead pipe" appearance. Post-inflammatory polyps may be present. The deposition of submucosal fat in the large bowel is found in up to $61 \%$ of cases and may result in additional wall thickening and, consequently, in luminal narrowing. As a result of inflammation, there may be proliferation of the pericolic/rectal fat, resulting in widening of the presacral space.

The most severe complication is the toxic megacolon, which appears in up to $5 \%$ of cases and carries the risk of perforation and peritonitis. The risk development of colorectal cancer increases with the extent and the duration of the disease [28, 29, 31]. 


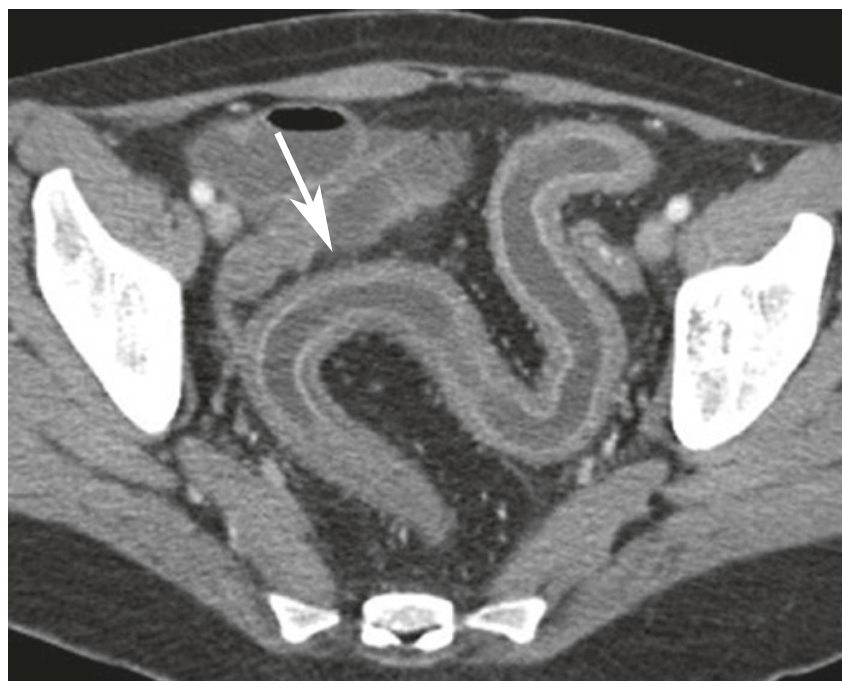

Fig. 19.9 Ulcerative colitis. The contrast-enhanced axial CT image shows continuous wall thickening of the sigmoid colon with mural stratification (arrow). Note that the outer contour of the colon is regular and smooth

\subsubsection{Crohn's Disease}

Crohn's disease may involve segments of the whole GI tract. However, Crohn's disease most often affects the terminal ileum and the proximal colon. Unlike ulcerative colitis, Crohn's disease typically affects the GI tract in a discontinuous way (so-called skip lesions), and the inflammatory process is transmural in nature.

CT usually misses the early stages of Crohn's disease. With progression of the disease, distinct mural thickening and luminal narrowing occur. In the acute stage of the disease, wall thickening presents with mural stratification mostly related to submucosal edema. The degree of contrast enhancement of the bowel wall correlates with the severity of the disease. Compared to ulcerative colitis, the outer contour of the colonic wall is irregular. As a result of the hyperemia from the inflammatory process, the local mesenteric vessels are dilated and widely spaced. This has been described as the "comb sign," indicating disease activity (Fig. 19.10). A progressive increase in higherdensity pericolic fat is called fibrofatty proliferation, resulting in separation of the bowel loops. Usually, multiple mesenteric lymph nodes, measuring $<10 \mathrm{~mm}$ in the short-axis diameter, are present. Extensive, intersecting linear transverse and longitudinal ulcerations can result in the so-called cobblestone pattern. With progression of the disease, the transmural inflammation is accompanied by irreversible fibrosis. On crosssectional imaging, this presents with a loss of mural stratification, with mural thickening and homogeneous attenuation [28, 29, 31].

Frequent complications are fistula, abscesses, adhesions, and stenosis, leading to bowel obstruction. Fistula can appear as ill-defined soft tissue bands extending either to another part of the gastrointestinal tract or to the skin. Abscesses are most

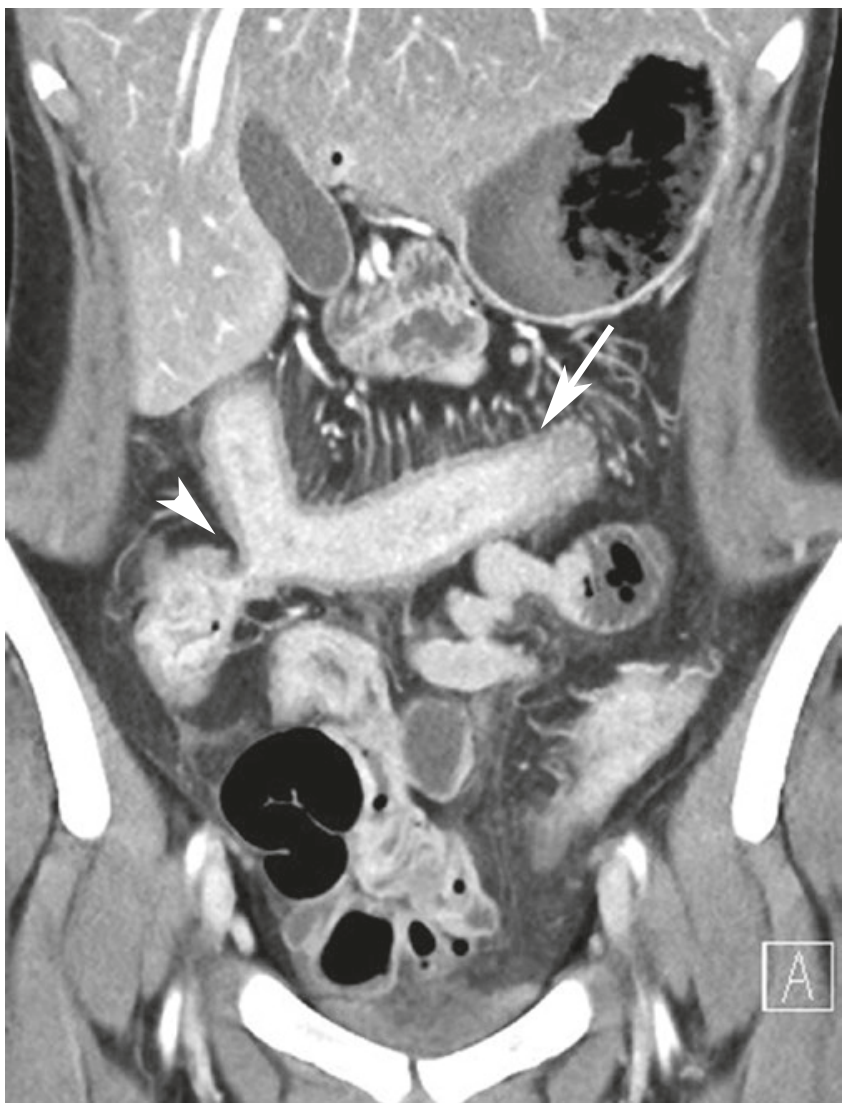

Fig. 19.10 Crohn's disease. The contrast-enhanced coronal CT image shows mural thickening of the transverse colon with dilated mesenteric vessels (comb sign) and fistula formation to the ascending colon (arrowhead). Note the inflammatory mural enhancement of small bowel loops

frequently associated with the penetrating type of Crohn's disease, affecting the small bowel with or without the colon. Stenosis in Crohn's disease shows, in many cases, circular, cone-shaped wall thickening with increased contrast enhancement and involvement of a longer segment. In other cases, short stenosis with wall thickening and abrupt shoulders at the proximal and distal end occur, which renders differentiation from malignant stenosis impossible. Perforations are uncommon and usually contained. Conglomerate masses are present if there is involvement of multiple bowel segments or a large bowel segment with fistulization and abscess formation. There is a slightly increased risk of developing colorectal cancer and lymphoma as a complication of the disease. These neoplasms mostly affect the small bowel. The presence of lymph node enlargement $>10 \mathrm{~mm}$ in the short-axis diameter should raise the suspicion of malignancy.

\subsubsection{Infectious Colitis}

Inflammation of the colon can be caused by bacterial, viral, fungal, or parasitic infection. The diagnosis of infectious 
colitis is readily achieved, based on the typical presentation of GI symptoms. Stool culture may be required to identify specific organisms. In general, most of these diseases are self-limiting, and dedicated imaging is not required for diagnosis. However, cross-sectional imaging may be required to assess the extent, the severity, and the potential complications of the disease.

Radiologic imaging features are not specific for a particular microorganism. Infectious colitis presents with diffuse colonic wall thickening, frequently with a so-called target appearance caused by mural stratification. This phenomenon is caused by mucosal CM enhancement and submucosal edema. Colonic air-fluid levels may be present. Extracolonic changes may include pericolonic fat stranding as well as ascites.

The affected colonic segment may suggest a specific organism. For instance, colonic infection with shigella or salmonella is limited to the right colon, while diffuse involvement may be seen with CMV and E. coli. Gonorrhea, herpes virus, and $C$. trachomatis typically involve the rectosigmoid area (Fig. 19.11) [32, 33].

\subsubsection{Pseudomembranous Colitis}

Pseudomembranous colitis (PMC) is an acute infectious colitis that results from an unopposed bacterial overgrowth of Clostridium difficile, typically following broad-spectrum antibiotic therapy or chemotherapy. Bacterial enterotoxins lead to superficial ulceration and subsequent formation of pseudomembranes. Often, the whole colon is involved; how- ever, both the rectosigmoid colon and the right colon may be affected in isolation.

Typical imaging findings include marked circumferential diffuse colonic wall thickening, caused by distinct mucosal and submucosal edema. The edema leads to a typical low attenuation aspect of the colonic wall. Wall thickening is more pronounced than in other types of colitis. After IV CM administration, mucosal and serosal enhancement reveals a target enhancement pattern, consisting of three concentric rings of different attenuation. Edematous thickening of the haustral outpouchings, as well as semilunar folds, leads to the appearance of an accordion on longitudinal crosssectional (accordion sign). Pericolonic changes include fat stranding and, often, ascites (Fig. 19.12). Potential complications include toxic megacolon and colonic perforation [34].

\subsubsection{Noninfectious Colitis}

This summarizes a colonic inflammatory involvement related to either a heterogeneous range of disorders or secondary to medical treatment.

\subsubsection{Ischemic Colitis}

Ischemic colitis is a disease of elderly patients with atherosclerotic disease, hypotension, or heart failure. Vascular insufficiency and ischemia can lead to a secondary inflammation of the colon. Low-flow states and nonocclusive vessel disease are

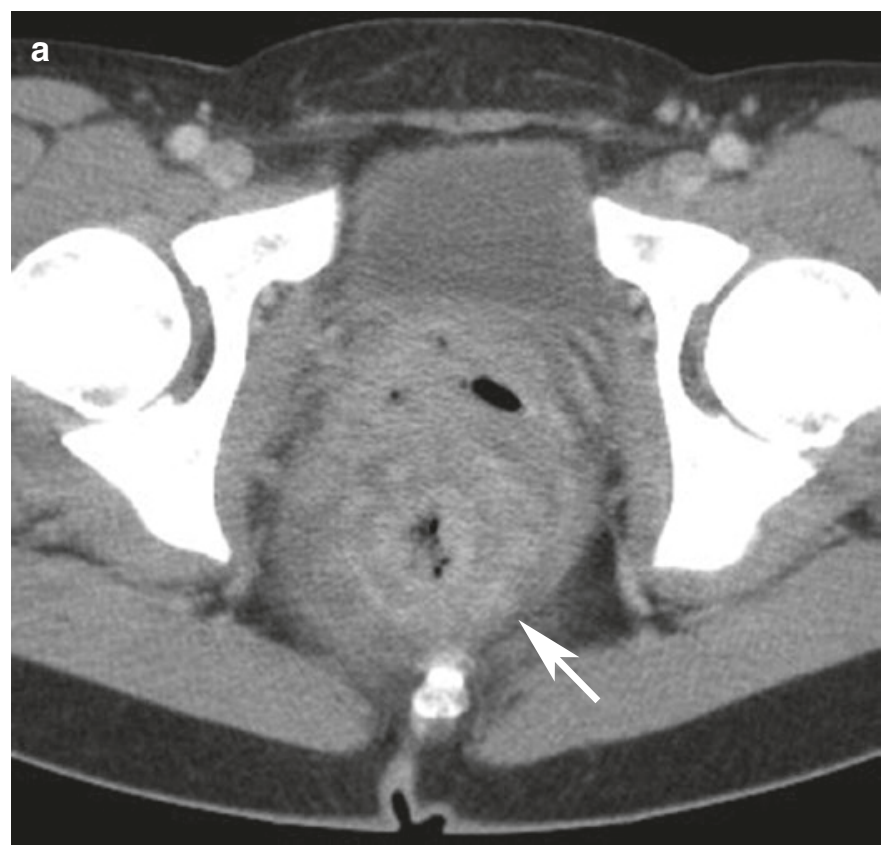

Fig. 19.11 (a, b) Rectal syphilis mimicking rectal cancer. (a) The contrast-enhanced axial CT image shows a large circumferential masslike rectal structure of with soft tissue density suggestive for a rectal

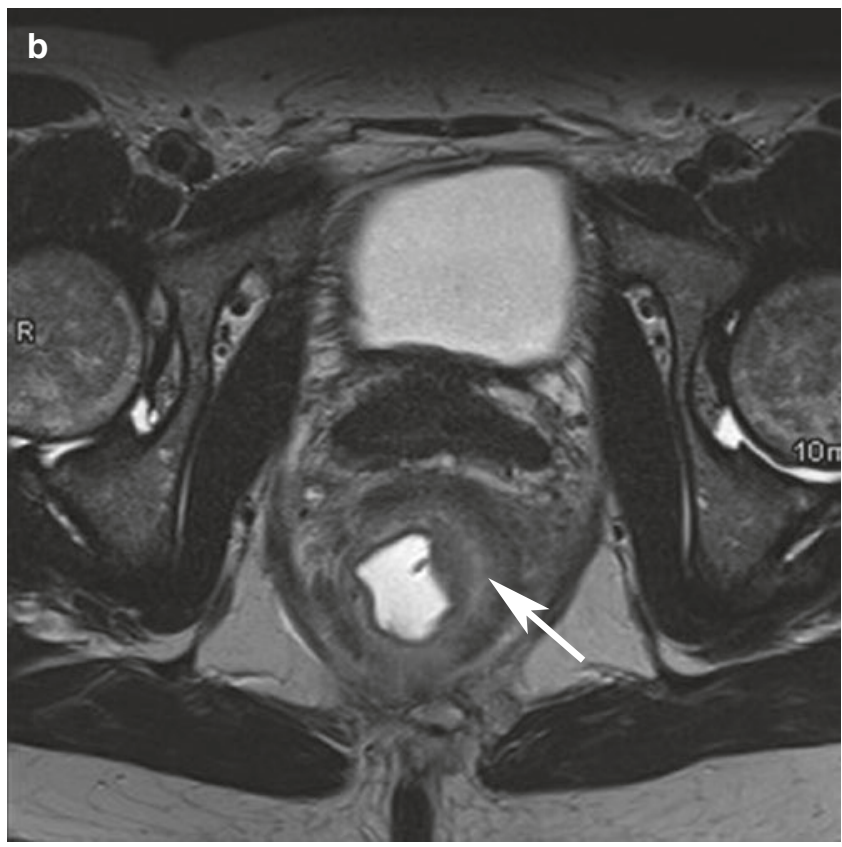

carcinoma (arrow). (b) Axial T2-weighted image of the rectum reveals a mural stratification caused by hyperintense, oedematous thickening of the submucosal layer, indicating an inflammatory process 


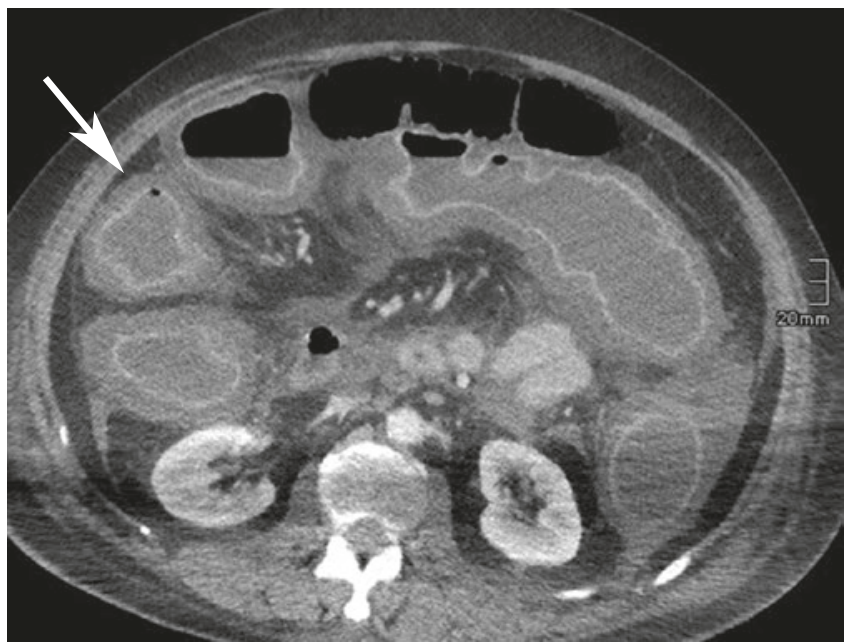

Fig. 19.12 Pseudomembranous colitis. The contrast-enhanced axial CT image shows marked mural thickening with intense enhancement of the mucosa and distinct submucosal edema of the entire colon (target sign) (arrow). Note the ascites

most common and typically lead to ischemic colitis in watershed areas at the splenic flexure and the rectosigmoid junction. The left colon is more frequently affected than the right colon. Imaging findings are nonspecific and may include mural thickening associated with submucosal edema. A target sign may be present. Following the acute event, fibrosis may lead to chronic strictures of the colonic lumen (Fig. 19.13) [29].

\subsubsection{Neutropenic Colitis}

Neutropenic colitis, also referred to as typhlitis, is a necrotizing inflammation of the cecum, ascending colon, and sometimes the terminal ileum seen in immunocompromised patients with severe neutropenia. It is caused by the transmural invasion of various microorganisms, including bacteria, virus, or fungi, without an inflammatory reaction. Imaging findings include a marked circumferential mural thickening of the right colon, typically the cecum, with distinct submucosal edema. Pneumatosis indicates the severity of the disease. Pericolonic fat stranding is a common finding. Complications include wall necrosis and perforation [29].

\subsubsection{Graft-Versus-Host Disease}

In graft-versus-host disease (GvHD), after allogenic bone marrow transplantation, donor lymphocytes attack the host tissues. GvHD is more frequently present in the small bowel but may also affect the colon. Imaging findings are nonspecific and include mural thickening associated with submucosal edema, which also affects mucosal enhancement (target sign). Typically, longer bowel segments are affected. Engorged mesenteric vascularization, fat stranding, and ascites may be present. In chronic stages, submucosal edema is replaced by fat tissue [35]. Clinical history leads to the specific diagnosis.

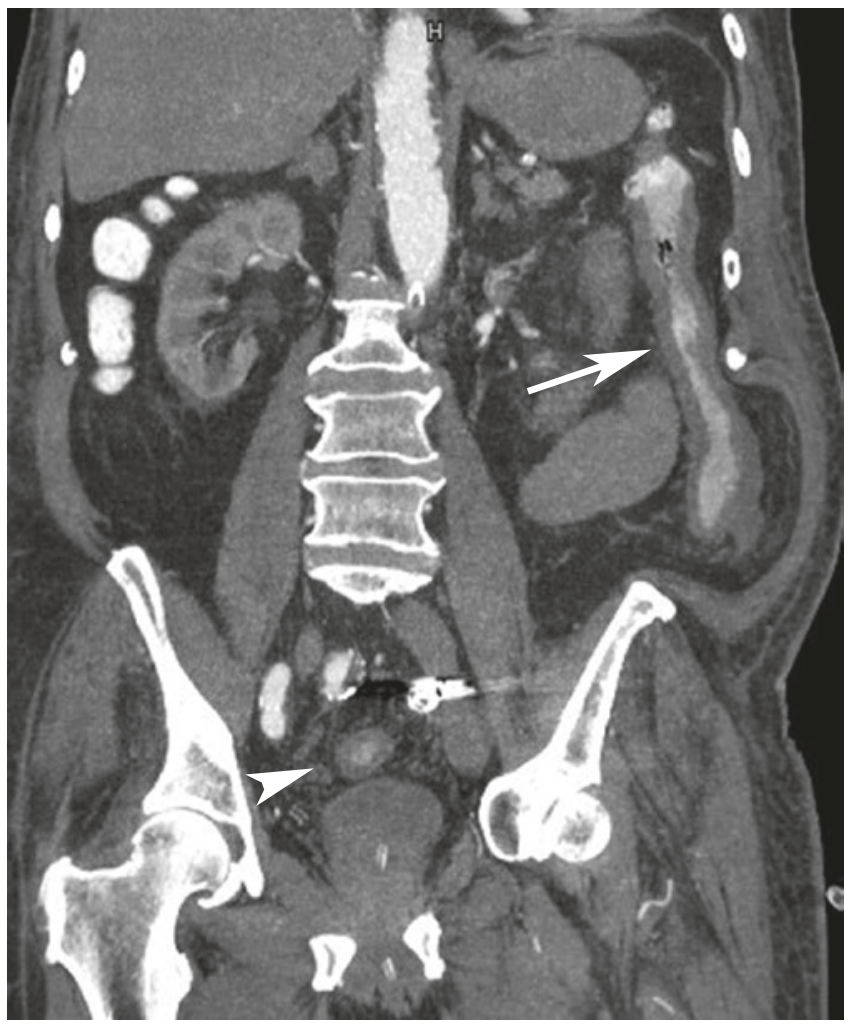

Fig. 19.13 Ischemic colitis. The contrast-enhanced axial CT image shows mural thickening of the descending (arrow) and sigmoid colon (arrowhead)

\subsubsection{Drug-Induced Colitis}

Drug-induced injury to the colon most frequently presents as colitis [36]. Cancer treatment with chemotherapeutics may lead neutropenic enterocolitis, pseudomembranous colitis, or pneumatosis intestinalis $[37,38]$. With the introduction of new anticancer drugs, such as checkpoint inhibitors, a new range of potential side effects from these therapies has been encountered. Checkpoint inhibitor-induced (e.g., ipilimumab-associated) colitis may occur in melanoma patients under immunotherapy (Fig. 19.14). On crosssectional imaging, it may present as mild, diffuse, bowelwall thickening, with fluid-filled colonic distention, and mesenteric vessel engorgement. It can also present as segmental colitis in the sigmoid colon in an area of pre-existing diffuse diverticulosis [39].

Long-term abuse of nonsteroidal anti-inflammatory drugs (NSAIDs) can induce colitis [40]. NSAID-induced colitis typically affects the right colon, with diffuse or segmental inflammation and ulcerations, as well as diaphragm-like fibrotic strictures. CT imaging findings are nonspecific, and wall thickening may simulate malignancy. Diaphragm-like strictures in the right colon on CTC may raise the suspicion for an inflammatory cause. A clinical history of NSAID abuse and an ischemic appearance at pathohistology leads to the diagnosis. 


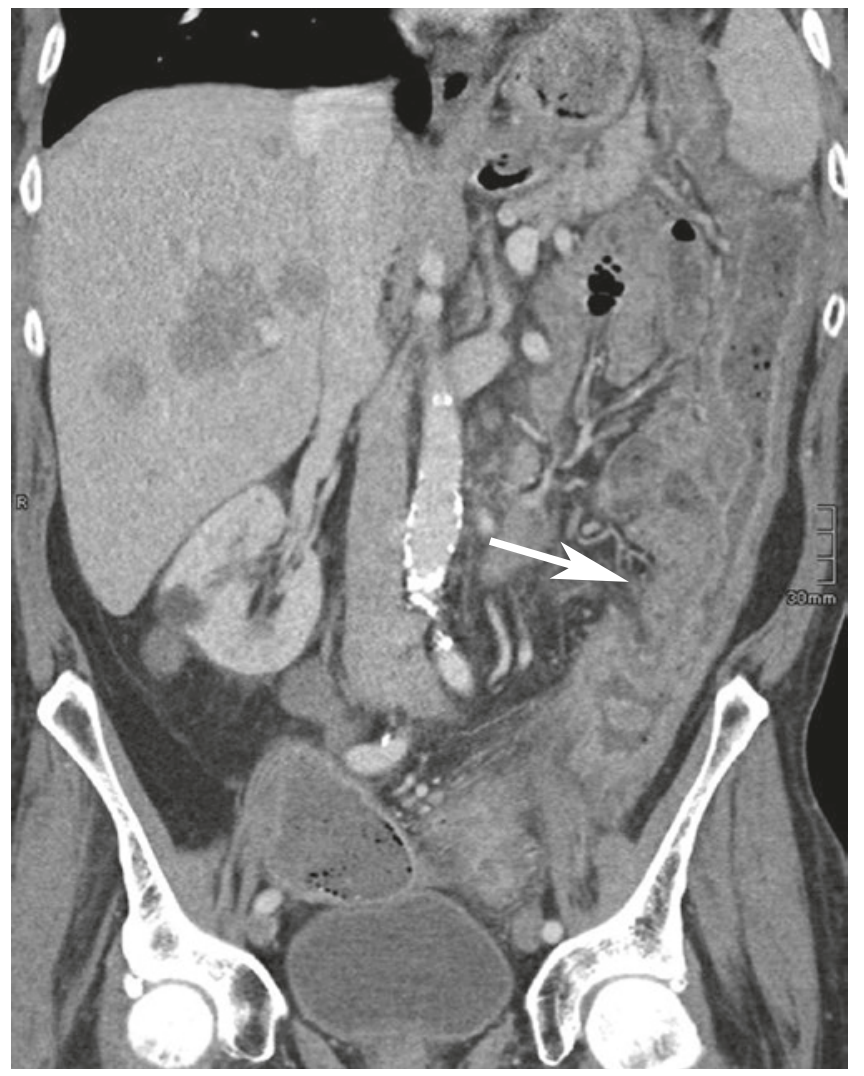

Fig. 19.14 Severe ipilimumab-associated colitis in a patient with metastatic melanoma. The contrast-enhanced coronal CT image shows marked thickening of the left colon with pericolic fat stranding and mesenteric vessel engorgement (arrow). Note the liver metastasis

\subsubsection{Radiation Colitis}

Since radiation therapy is often given for pelvic disease, the sigmoid colon and rectum are most commonly affected. Acute radiation injury, within 8 weeks after treatment, is typically self-limited and treated symptomatically. It is diagnosed clinically and does not require imaging. Imaging findings include nonspecific wall thickening and inflammatory stranding in the affected segments. In chronic radiation injury, 6-12 months after treatment, which results from radiation-induced endarteritis, smooth wall thickening and fibrosis may narrow the colonic lumen. Strictures and fistulas are possible complications. Increased pelvic fat may be present $[29,37]$.

\subsubsection{Epiploic Appendagitis}

Epiploic appendagitis is an uncommon inflammatory/ischemic process that results from torsion with infarction or spontaneous venous thrombosis of the appendices epiploicae. Patients may present clinically with sudden, focal abdominal pain, indistinguishable from diverticulitis or appendicitis. CT is diagnostic, typically showing an ovoid

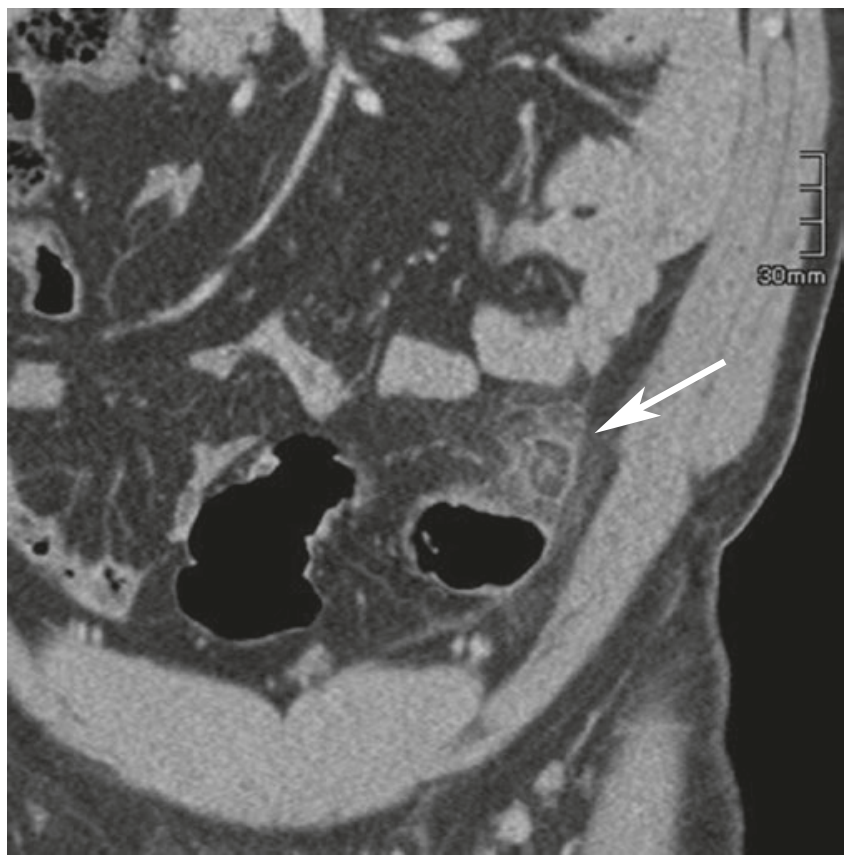

Fig. 19.15 Epiploic appendagitis. The contrast-enhanced coronal CT image shows a round fat containing pericolic lesion with stranding of the surrounding fat tissue (arrow). A central hyperdense "dot" within the lesion corresponds to the thrombosed vein

$1.5-3.5 \mathrm{~cm}$ fat-containing pericolic lesion with stranding of the surrounding fat tissue (Fig. 19.15). A central hyperdense "dot" can be identified within the lesion that corresponds to the thrombosed vein. Chronically, an infarcted epiploic appendix may calcify. Epiploic appendagitis is a self-limiting condition and does not require invasive intervention [41].

\section{Take Home Messages}

- Cross-sectional imaging techniques have become the most important radiologic tool for the evaluation of diseases of the colon and rectum.

- CTC is a reliable technique for the detection and classification of polypoid and stenotic lesions of the colon.

- At CTC, the most important imaging features to characterize a finding are morphology, internal structure and attenuation characteristics, and mobility. Inflammatory conditions of the colon represent a heterogeneous spectrum of diseases, showing, however, a considerable overlap of their imaging criteria.

- Radiologic differentiating features at standard cross-sectional imaging include the primary location within the GI tract, length of segmental involvement, degree of wall thickening, mural enhancement pattern, pericolonic and extraintestinal findings. 


\section{References}

1. Kim DH, et al. CT colonography versus colonoscopy for the detection of advanced neoplasia. N Engl J Med. 2007;357(14):1403-12.

2. Halligan $S$, et al. Computed tomographic colonography versus barium enema for diagnosis of colorectal cancer or large polyps in symptomatic patients (SIGGAR): a multicentre randomised trial. Lancet. 2013;381(9873):1185-93.

3. Spada C, et al. Clinical indications for computed tomographic colonography: European Society of Gastrointestinal Endoscopy (ESGE) and European Society of Gastrointestinal and Abdominal Radiology (ESGAR) guideline. Eur Radiol. 2015;25(2):331-45.

4. Endoscopic Classification Review, G. Update on the paris classification of superficial neoplastic lesions in the digestive tract. Endoscopy. 2005;37(6):570-8.

5. Mang T, et al. Evaluation of colonic lesions and pitfalls in CT colonography: a systematic approach based on morphology, attenuation and mobility. Eur J Radiol. 2013;82(8):1177-86.

6. Neri E, et al. The second ESGAR consensus statement on CT colonography. Eur Radiol. 2013;23(3):720-9.

7. Kim DH, et al. Contrast coating for the surface of flat polyps at CT colonography: a marker for detection. Eur Radiol. 2014;24(4):940-6.

8. Kudo S, et al. Nonpolypoid neoplastic lesions of the colorectal mucosa. Gastrointest Endosc. 2008;68(4 Suppl):S3-47.

9. Pickhardt PJ, et al. Carpet lesions detected at CT colonography: clinical, imaging, and pathologic features. Radiology. 2014;270(2):435-43.

10. Pickhardt PJ, et al. Evaluation of submucosal lesions of the large intestine: part 1. Neoplasms. Radiographics. 2007;27(6):1681-92.

11. Pickhardt PJ, et al. Evaluation of submucosal lesions of the large intestine: part 2. Non neoplastic causes. Radiographics. 2007;27(6):1693-703.

12. Jeong SY, et al. The usefulness of computed tomographic colonography for evaluation of deep infiltrating endometriosis: comparison with magnetic resonance imaging. J Comput Assist Tomogr. 2013;37(5):809-14.

13. Lefere P, Gryspeerdt S. CT colonography: avoiding traps and pitfalls. Insights Imaging. 2011;2(1):57-68.

14. Dachman AH, et al. CT colonography: visualization methods, interpretation, and pitfalls. Radiol Clin N Am. 2007;45(2):347-59.

15. Silva AC, et al. Spectrum of normal and abnormal CT appearances of the ileocecal valve and cecum with endoscopic and surgical correlation. Radiographics. 2007;27(4):1039-54.

16. Mang T, Schima W. CT colonography: a guide for clinical practice. 1st ed. Stuttgart: Georg Thieme Verlag KG; 2013.

17. Regge $\mathrm{D}$, et al. Ileocecal valve imaging on computed tomographic colonography. Abdom Imaging. 2005;30(1):20-5.

18. Pickhardt PJ, et al. Primary neoplasms of the appendix: radiologic spectrum of disease with pathologic correlation. Radiographics. 2003;23(3):645-62.

19. Lange N, Barlow D, Long J. Mucocele of the appendix on screening CT colonography: a case report. Abdom Imaging. 2008;33(3):267-9.
20. Johnson EK, Arcila ME, Steele SR. Appendiceal inversion: a diagnostic and therapeutic dilemma. JSLS. 2009;13(1):92-5.

21. Lefere P, et al. Diverticular disease in CT colonography. Eur Radiol. 2003;13(Suppl 4):L62-74.

22. Mang T, et al. Pitfalls in multi-detector row CT colonography: a systematic approach. Radiographics. 2007;27(2):431-54.

23. Gryspeerdt S, Lefere P. Chronic diverticulitis vs. colorectal cancer: findings on CT colonography. Abdom Imaging. 2012;37(6):1101-9.

24. Lips LM, et al. Sigmoid cancer versus chronic diverticular disease: differentiating features at CT colonography. Radiology. 2015;275(1):127-35.

25. Choi YJ, et al. CT colonography for follow-up after surgery for colorectal cancer. AJR Am J Roentgenol. 2007;189(2):283-9.

26. Macari M, Balthazar EJ. CT of bowel wall thickening: significance and pitfalls of interpretation. AJR Am J Roentgenol. 2001;176(5):1105-16.

27. Wittenberg J, et al. Algorithmic approach to CT diagnosis of the abnormal bowel wall. Radiographics. 2002;22(5):1093-107; discussion 1107-9.

28. Mang T, et al. Virtual colonoscopy: beyond polyp detection, in virtual colonoscopy: a practical guide. Berlin: Springer; 2009. p. 199-217.

29. Horton KM, Corl FM, Fishman EK. CT evaluation of the colon: inflammatory disease. Radiographics. 2000;20(2):399-418.

30. Chintapalli KN, et al. Diverticulitis versus colon cancer: differentiation with helical CT findings. Radiology. 1999;210(2):429-35.

31. Gore RM, et al. CT features of ulcerative colitis and Crohn's disease. AJR Am J Roentgenol. 1996;167(1):3-15.

32. Thoeni RF, Cello JP. CT imaging of colitis. Radiology. 2006;240(3):623-38.

33. Cha JM, Choi SI, Lee JI. Rectal syphilis mimicking rectal cancer. Yonsei Med J. 2010;51(2):276-8.

34. Kawamoto S, Horton KM, Fishman EK. Pseudomembranous colitis: spectrum of imaging findings with clinical and pathologic correlation. Radiographics. 1999;19(4):887-97.

35. del Campo L, et al. Abdominal complications following hematopoietic stem cell transplantation. Radiographics. 2014;34(2):396-412.

36. McGettigan MJ, et al. Imaging of drug-induced complications in the gastrointestinal system. Radiographics. 2016;36(1):71-87.

37. Viswanathan $\mathrm{C}$, et al. Imaging of complications of oncological therapy in the gastrointestinal system. Cancer Imaging. 2012;12:163-72.

38. Torrisi JM, et al. CT findings of chemotherapy-induced toxicity: what radiologists need to know about the clinical and radiologic manifestations of chemotherapy toxicity. Radiology. 2011;258(1):41-56.

39. Kim KW, et al. Ipilimumab-associated colitis: CT findings. AJR Am J Roentgenol. 2013;200(5):W468-74.

40. Klein M, Linnemann D, Rosenberg J. Non-steroidal antiinflammatory drug-induced colopathy. BMJ Case Rep. 2011;2011:bcr1020103436.

41. Singh AK, et al. Acute epiploic appendagitis and its mimics. Radiographics. 2005;25(6):1521-34.

Open Access This chapter is licensed under the terms of the Creative Commons Attribution 4.0 International License (http://creativecommons. org/licenses/by/4.0/), which permits use, sharing, adaptation, distribution and reproduction in any medium or format, as long as you give appropriate credit to the original author(s) and the source, provide a link to the Creative Commons license and indicate if changes were made.

The images or other third party material in this book are included in the book's Creative Commons license, unless indicated otherwise in a credit line to the material. If material is not included in the book's Creative Commons license and your intended use is not permitted by statutory regulation or exceeds the permitted use, you will need to obtain permission directly from the copyright holder. 